\title{
Enhanced Imaging Colonoscopy Facilitates Dense Motion-Based 3D Reconstruction
}

\author{
Pablo F. Alcantarilla ${ }^{1}$, Adrien Bartoli ${ }^{2}$, François Chadebecq ${ }^{2,3}$, Christophe Tilmant ${ }^{3}$ and Vincent Lepilliez $^{4}$
}

\begin{abstract}
We propose a novel approach for estimating a dense 3D model of neoplasia in colonoscopy using enhanced imaging endoscopy modalities. Estimating a dense 3D model of neoplasia is important to make 3D measurements and to classify the superficial lesions in standard frameworks such as the Paris classification. However, it is challenging to obtain decent dense 3D models using computer vision techniques such as Structurefrom-Motion due to the lack of texture in conventional (white light) colonoscopy. Therefore, we propose to use enhanced imaging endoscopy modalities such as Narrow Band Imaging and chromoendoscopy to facilitate the $3 \mathrm{D}$ reconstruction process. Thanks to the use of these enhanced endoscopy techniques, visualization is improved, resulting in more reliable feature tracks and 3D reconstruction results. We first build a sparse 3D model of neoplasia using Structure-from-Motion from enhanced endoscopy imagery. Then, the sparse reconstruction is densified using a Multi-View Stereo approach, and finally the dense 3D point cloud is transformed into a mesh by means of Poisson surface reconstruction. The obtained dense 3D models facilitate classification of neoplasia in the Paris classification, in which the 3D size and the shape of the neoplasia play a major role in the diagnosis.
\end{abstract}

Index Terms-Narrow Band Imaging, Chromoendoscopy, Structure-from-Motion, Multi-View Stereo, Poisson Surface Reconstruction, Paris classification.

\section{INTRODUCTION}

Colorectal cancer is one of the leading causes of cancerrelated deaths and one of the most common cancer in men and women in the world. Early diagnosis greatly impacts colorectal cancer survival rates. One of the most extended modalities to facilitate early diagnosis is colonoscopy [1], in which an endoscope is introduced through the rectum and the colon. Physicians can use the endoscope video stream to detect and identify tissue abnormalities such as neoplasia (an abnormal growth of tissue).

Standard (white light) colonoscopy is the most widely used and preferred colorectal cancer screening technique[2]. However, substantial miss rates for adenoma and cancers have been reported with standard colonoscopy. Therefore, enhanced colonoscopy techniques such as Narrow Band Imaging (NBI) and chromoscopy have been widely used recently to improve visualization and colorectal cancer diagnosis [3], [4]. NBI [5] is a high-resolution endoscopic

${ }^{1} \mathrm{P}$. F. Alcantarilla is with the School of Interactive Computing, Georgia Institute of Technology, Atlanta, USA

${ }^{2} \mathrm{~A}$. Bartoli and F. Chadebecq are with ISIT UMR $6284 \mathrm{CNRS/Université}$ d'Auvergne, Clermont-Ferrand, France

${ }^{3}$ C. Tilmant and F. Chadebecq are with the Institute Pascal UMR 6602 CNRS/UBP/IFMA, Clermont-Ferrand, France

${ }^{4} \mathrm{~V}$. Lepilliez is with the Gastroenterology Department, Hôpital Edouard Herriot, Lyon, France technique that improves visualization by exploiting the tissue absorption linked to the wavelenght of the light, enhancing the fine structure of the mucosal surface and the microvascular and microstructural pit patterns. On the other hand, chromoscopy [6] applies contrast dyes such as indigo carmine to improve the detection of mucosal abnormalities. Chromoscopic agents permit early detection of neoplastic colorectal lesions, in particular flat and depressed types [7]. In general, chromoscopy is a time consuming preparation due to the application of the staining agent, whereas in NBI no preparation is needed due to the modification of the endoscopic system.

The diagnosis of neoplasia relies on a general framework for the endoscopic classification of superficial lesions known as the Paris classification [8]. In this classification, neoplasias are classified into different levels according to its size, shape and depth of invasion into the submucosa. This classification directly influences the decision of resection. Within this context, we think it is of great interest to develop non-invasive computer vision techniques that can obtain a dense 3D model of neoplasias and therefore provide more feature to apply the Paris classification.

Computer vision techniques such as Structure-fromMotion (SfM) [9] seem to be an interesting approach for diagnosis in colonoscopy. However, it is challenging to use SfM approaches in standard colonoscopy due to the lack of texture and the paucity of reliable 2D features to be tracked over several frames. To overcome this limitation we can take advantage of enhanced colonoscopy techniques such NBI and chromoscopy. In both cases, the enhanced visualization and pit patterns provide reliable feature tracks that can be used efficiently in SfM. Figure 1 depicts an example of a 3D neoplasia reconstruction with standard colonoscopy and with chromoscopy.

In this paper we propose an off-line method that uses SfM to build an initial sparse 3D model of neoplasia from enhanced colonoscopy imagery. Then, the initial 3D model is densified by means of Multi-View Stereo (MVS) techniques [10]. The resulting dense 3D point cloud is then transformed into a dense 3D mesh by using Poisson surface reconstruction [11]. The final dense 3D model can be used by the physicians for classifying neoplasias according to Paris classification and only then making the decision of resecting it or not. The rest of the paper is organised as follows: we describe related work in Section II and briefly review the Paris classification in Section III. Our dense 3D modeling of neoplasia is introduced in Section IV. Experimental results 


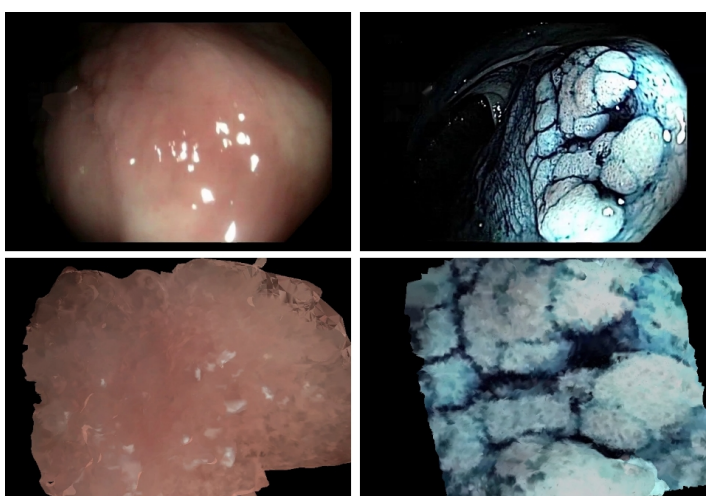

Fig. 1. 3D Neoplasia reconstruction results. First column: standard colonoscopy. Second column: enhanced colonoscopy. First row: sample input image. Second row: 3D view from the dense model. It can be observed that the 3D model with standard colonoscopy cannot recover accurately the 3D geometry of the neoplasia due to the lack of texture. However, when using enhanced colonoscopy the 3D model is recovered more accurately.

are reported in Section V. Final conclusions and future work are described in Section VI.

\section{RELATED WORK}

Computer vision techniques that recover the 3D geometry of a rigid scene have been proposed in different medical imaging applications. These techniques use an SfM pipeline to recover a sparse 3D geometry by either using monocular [12], [13] or stereo imaging devices [14]. The main problem with monocular frameworks is that the 3D geometry of the scene can be recovered up to a scale factor, while in the stereo case this ambiguity does not occur. In addition, sparse 3D models do not provide enough information to be interpreted by physicians.

In colonoscopy, there have been some approaches focused more on automatic segmentation and classification of neoplasias based on 2D appearance models [15], [16]. While this is an interesting and challenging problem, the gold standard for the endoscopic classification of superficial lesions is the Paris classification, in which neoplasias are classified into different types of lesions according to their 3D size and shape. Therefore, we think that an approach that can estimate the size of neoplasias in $3 \mathrm{D}$ is more interesting than 2D segmentation approaches that do not recover the underlying 3D geometry. SfM in standard colonoscopy was tried before in [12]. However, the main flaw of this approach is that it cannot recover accurately the 3D surface due to the lack of texture and the sparse number of points.

Recently, Chadebecq et al. [17] proposed an image-based method to estimate the size of the neoplasias combining Depth-From-Focus (DFF) and Depth-From-Defocus (DFD) techniques [18], [19]. Even though, this method is efficient for recovering the absolute scale by automatically detecting the blur-unblur breakpoint in a colonoscopy video sequence, it is not reliable for estimating a dense 3D model of a neoplasia. This is because the method assumes that a neoplasia is frontoparallel to the gatroscope's tip (which is a strong assumption that is not true in most of the scenarios) yielding an underestimated size.

\section{THE PARIS ClASSIFICATION}

In this section, we briefly review the Paris classifcation for neoplastic lesions in colonoscopy [8]. In the Paris classification, neoplastic lesions with superficial morphology are classified as Type-0. Other more advanced gastric tumours are classified in different types. Neoplastic lessions are those whose endoscopic appearance suggests that the depth of penetration in the digestive wall is not more than into the submucosa. Depending on the size and shape, Type- 0 neoplastic lesions are classified into different sublevels:

- 0-I: Polypoid, pedunculated or sessile

- 0-IIa: Non-Polypoid, superficial or elevated

- 0-IIb: Non-Polypoid, flat

- 0-IIc: Non-Polypoid, superficial shallow, depressed

- 0-III: Non-Polypoid, excavated

\section{Dense 3D Neoplasia Modeling}

Given an enhanced colonoscopy video stream with NBI or chromoscopy settings the user chooses one frame from which the $3 \mathrm{D}$ reconstruction starts. Posterior keyframes are selected automatically from the video stream considering that we have enough motion and feature correspondences between each pair of selected frames. Figure 2 depicts an overall overview of the different reconstruction steps in our approach.

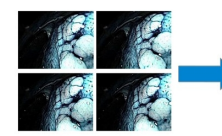

Enhanced

Colonoscopy

Images

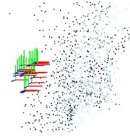

Rigid SfM

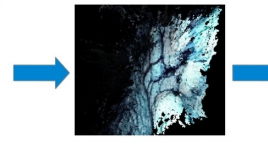

MVS

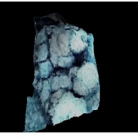

Poisson Rect and Scale
Fig. 2. Different steps in our dense 3D neoplasia modeling.

Firstly, the endoscope is calibrated in a setup process to estimate camera's intrinsics [20]. We then perform radial distortion correction which is usually large in colonoscopy imagery. Considering that the camera's intrinsics are known, we first perform SfM on a set of selected keyframes with enough baseline between them. The goal of SfM algorithms is to reconstruct an unknown $3 \mathrm{D}$ scene and camera motion from a set of feature correspondences. We use a similar SfM pipeline as the one described in [21], using the Speeded Up Robust Features (SURF) [22] as our feature detector and descriptor. We avoid detecting features in areas where we have specular reflectance by detecting saturated blobs in the image and use these blobs as an input mask for the feature detector algorithm. Once features are detected in every keyframe, we match feature descriptors between the image pairs using the nearest neighbor distance ratio strategy as described in [23]. Then, we identify the inliers from the set of putative matches by fitting a Fundamental matrix in a RANSAC procedure [24]. 
The output of the SfM pipeline is a sparse 3D reconstruction of a rigid scene and the camera motion (rotation and translation) for each keyframe. This kind of 3D model is still not useful enough to measure the size of neoplasia due to the small number of 3D points. Therefore, we run a model densification step. We use the MVS technique described in [10] to obtain a dense 3D point cloud from the optimized camera poses. This technique reconstructs a set of oriented patches covering the surface of an object of interest by minimizing a photometric discrepancy function. Then, the dense 3D point cloud is converted to a dense mesh by using Poisson surface reconstruction [11].

At this point we have a dense 3D mesh of a surface up to a scale factor. In order to recover the correct scale of the 3D model, we use the method described in [17]. By combining DFF and DFD techniques and finding the blur-unblur breakpoint in the analyzed video sequence this method is able to obtain the correct scale of the 3D model.

\section{RESULTS}

In order to validate our approach, we show experimental resuls on several in-vivo sequences. We show quantitative results on sequences with different types of neoplasias. All the sequences were acquired with the Olympus HQ-190 endoscope. We consider several chromoscopy sequences and one NBI experiment.

Chromoscopy and NBI have an extreme importance in the quality of the dense 3D model reconstruction, since SfM and MVS methods are prone to failure when reconstructing textureless surfaces. Dense models cannot be obtained with normal endoscope settings. Figure 3(a) depicts the detected SURF features using standard colonoscopy, whereas (b) depicts the detected features using enhanced colonoscopy (chromoscopy) for the same surface. For both images we used the same detector response threshold. As can be observed, the texture of the underlying surface is highlighted when using enhanced colonoscopy and thus the number of detected features is much higher than in the standard colonoscopy case.

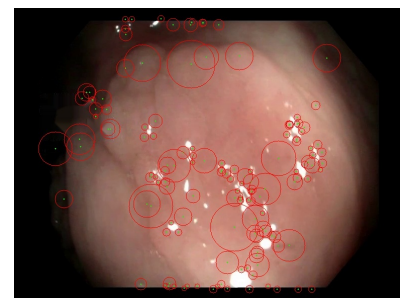

(a)

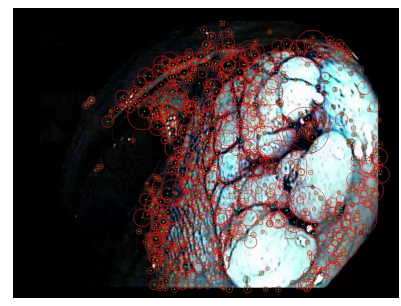

(b)
Fig. 3. Number of detected features per image: (a) 138 features using standard colonoscopy (b) 1069 features using enhanced colonoscopy. The features are depicted in red circles with radius proportional to the scale of the detected keypoint.

Figure 4 shows 3D reconstruction results for five different experiments. For each experiment we show a curve surrounding the neoplasia of interest in red, highlighting the superficial lesion for which we obtained size estimates.
Table I shows size estimation and Paris classification results for the different experiments. We compute the diameter of the different neoplasias, depicted by a red line as shown in Figure 4. The first experiment shows an adenoma whith a sessile polyp. The second one consists of a laterally spreading tumor in the colon. The third experiment consists of two main pendunculated hyperplasias. The fourth one shows a laterally spreading adenoma in the colon, whereas the fifth experiment consists on two sessile polyps.

\begin{tabular}{|c|c|c|}
\hline Exp. & Diameter (mm) & Paris Cl. \\
\hline 1 & 3.32 & 0-IIa \\
\hline 2 & 7.29 & 0-IIa \\
\hline 3 & 2.87 & 0-Ip \\
\hline 4 & 0.82 & 0-IIc \\
\hline 5 & 2.91 & 0-IIa \\
\hline
\end{tabular}

TABLE I

SIZE ESTIMATION OF NEOPLASIAS AND PARIS CLASSIFICATION RESULTS.

\section{CONClusions And Future Work}

In this paper we have proposed an off-line method for building a dense 3D model of neoplasia using enhanced colonoscopy (NBI and chromoscopy). The use of enhanced colonoscopy facilitates SfM approaches to obtain reliable $3 \mathrm{D}$ reconstruction results due to the improved texture. To the best of our knowledge, this is the first work to propose SfM in enhanced colonoscopy obtainind reliable 3D dense neoplasia reconstructions. We believe this is an important contribution since previous approaches that use SfM in standard colonoscopy are not able to estimate a reliable 3D surface due to the lack of texture and paucity of reliable features. The experimental results are encouraging and show that the proposed approach can be of benefit to physicians in order to facilitate the diagnosis of neoplasia according to the Paris classification. In the near future, we are interested in performing an extensive evaluation with ground-truth data and developping a real-time system based on the proposed techniques with automatic classification of neoplasia that can be used by physicians during exploration.

\section{ACKNOWLEDGMENTS}

The authors would like to acknowledge the support of ANR through project SYSEO.

\section{REFERENCES}

[1] J. P. Hassinger, S. D. Holubar, R. Pendlimari, E. J. Dozois, D. W. Larson, and R. R. Cima, "Effectiveness of a multimedia-based educational intervention for improving colon cancer literacy in screening colonoscopy patients," Diseases of the Colon \& Rectum, vol. 53, no. 9 , pp. 1301-1307, 2010.

[2] D. K. Rex, D. A. Johnson, J. C. Anderson, P. S. Schoenfeld, C. A Burke, and J. M. Inadomi, "American college of gastroenterology guidelines for colorectal cancer screening," Am. J. Gastroenterology, vol. 104, no. 3, pp. 739-750, 2009.

[3] H. Tajiri and H. Niwa, "Recent advances in electronic endoscopes: Image-enhanced endoscopy," Japan Gastroenterological Endoscopy Society, vol. 51, no. 3, pp. 199-203, 2008. 

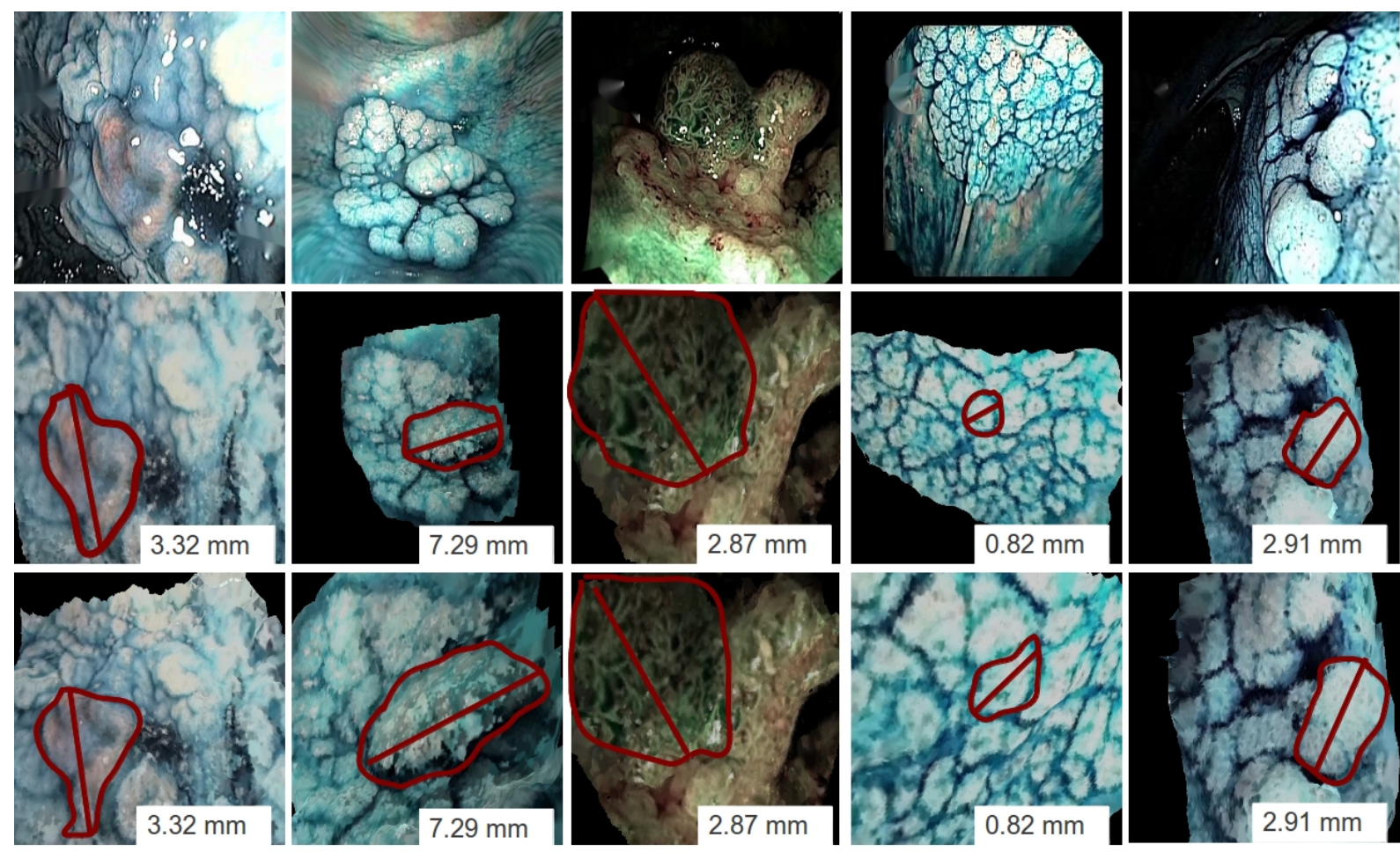

(1)

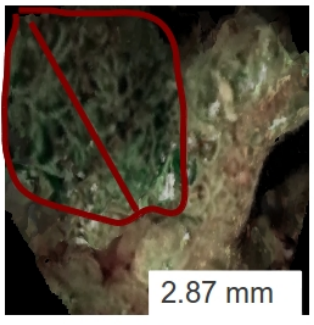

(3)

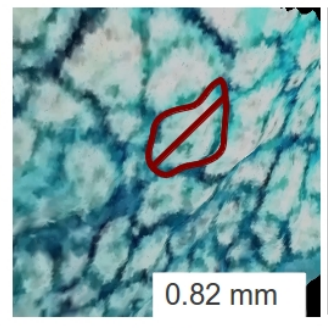

(4)

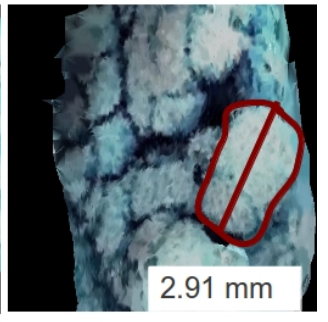

(5)

Fig. 4. Dense 3D neoplasia reconstruction results: Each column depicts results for a different experiment. First row: sample input image. Second and Third row: different viewpoints from the 3D model. Experiments 1,2,4,5 use chromoscopy with indigo carmine as the contrast dye. Experiment 3 uses NBI.

[4] J. P. Nass and S. E. Conolly, "Current status of chromoendoscopy and narrow band imaging in colonoscopy," Clin. Colon Rectal Surg., vol. 23, no. 1, pp. 21-30, 2010 .

[5] R. Singh, V. Owen, A. Shonde, P. Kaye, C. Hawkey, and K. Ragunath, "White light endoscopy, narrow band imaging and chromoendoscopy with magnification in diagnosing colorectal neoplasia," World Journal of Gastrointestinal Endocopy, vol. 1, no. 1, pp. 45-50, 2009.

[6] M. Tada, S. Katoh, Y. Kohli, and K. Kawai, "On the dye spraying method in colonofiberscopy," Endoscopy, vol. 8, no. 2, pp. 70-74, 1977.

[7] D. P. Hurlstone and D. S. Sanders, "Recent advances in chromoscopic colonoscopy and endomicroscopy," Current Gastroenterology Reports, vol. 8, no. 5, pp. 409-415, 2006.

[8] Endoscopic Classification Review Group, "Update on the Paris classification of superficial neoplastic lesions in the digestive tract," Endoscopy, vol. 37, no. 6, pp. 570-578, 2005.

[9] R. Hartley and A. Zisserman, Multiple View Geometry in Computer Vision, 2nd ed. Cambridge University Press, ISBN: 0521540518, 2004.

[10] Y. Furukawa and J. Ponce, "Accurate, dense, and robust multiview stereopsis," IEEE Trans. Pattern Anal. Machine Intell., vol. 32, no. 8, pp. 1362-1376, 2010.

[11] M. Kazhdan, M. Bolitho, and H. Hoppe, "Poisson surface reconstruction," in Symposium on Geometry Processing, 2006.

[12] T. Thormählen, H. Broszio, and P. N. Meier, "Three-dimensional endoscopy," in Falk Symposium No. 124, Medical Imaging in Gastroenterology and Hepatology, Hannover, Germany, 2001.

[13] O. G. Grasa, J. Civera, and J. M. M. Montiel, "EKF Monocular SLAM with Relocalization for Laparoscopic Sequences," in IEEE Intl. Conf. on Robotics and Automation (ICRA), Shanghai, China, 2011.

[14] P. Mountney, D. Stoyanov, A. J. Davison, and G. Z. Yang, "Simultaneous stereoscope localization and soft-tissue mapping for minimally invasive surgery," in Medical Image Computing and Computer Assisted Intervention (MICCAI), 2006.

[15] T. Stehle, R. Auer, S. Gross, A. Bahrens, J. Wulff, T. Aach, R. Winograd, C. Trautwein, and J. Tischendorf, "Classification of colon polyps in NBI endoscopy using vascularization features," in Medical Imaging: Computer-Aided Diagnosis, 2009.

[16] J. Bernal, F. Sánchez, and F. Vilariño, "Towards automatic polyp detection with a polyp appearance model," Pattern Recognition, vol. 45 , no. 9, pp. 3166-3182, 2012.

[17] F. Chadebecq, C. Tilmant, and A. Bartoli, "Measuring the size of neoplasia in colonoscopy using depth-from-defocus," in IEEE Intl. Engineering in Medicine and Biology Society (EMBC), San Diego, California, USA, 2012

[18] S. K. Nayar and Y. Nakagawa, "Shape from focus," IEEE Trans. Pattern Anal. Machine Intell., vol. 16, no. 8, pp. 824-831, 1994.

[19] P. Favaro and S. Soatto, "A geometric approach to shape from defocus," IEEE Trans. Pattern Anal. Machine Intell., vol. 27, no. 3, pp. 406-417, 2005.

[20] J. Bouguet, "Documentation: Camera Calibration Toolbox for Matlab,' 2008. [Online]. Available: www.vision.caltech.edu/bouguetj/calib_doc/

[21] N. Snavely, S. S. Seitz, and R. Szeliski, "Modeling the world from Internet photo collections," Intl. J. of Computer Vision, vol. 80, no. 2, pp. 189-210, 2008.

[22] H. Bay, A. Ess, T. Tuytelaars, and L. V. Gool, "SURF: Speeded up robust features," Computer Vision and Image Understanding, vol. 110, no. 3, pp. 346-359, 2008.

[23] D. G. Lowe, "Distinctive image features from scale-invariant keypoints," Intl. J. of Computer Vision, vol. 60, no. 2, pp. 91-110, 2004

[24] R. Bolles and M. Fischler, "A RANSAC-based approach to model fitting and its application to finding cylinders in range data," in Intl. Joint Conf. on AI (IJCAI), Vancouver, Canada, 1981, pp. 637-643. 